\title{
BMJ Open Urinary placental growth factor as a predictor of complications in hypertensive disorders in pregnancy: a protocol for systematic review and meta- analysis
}

\author{
Camille Francoeur, ${ }^{1}$ Julie Moreau, ${ }^{2}$ Samuel Lemaire-Paquette, ${ }^{2}$ \\ Marie-Claude Battista, ${ }^{3}$ Marie-Eve Roy-Lacroix, ${ }^{4}$ Anne-Marie Côté (1) ${ }^{1,2}$
}

To cite: Francoeur C, Moreau J, Lemaire-Paquette $\mathrm{S}$, et al. Urinary placental growth factor as a predictor of complications in hypertensive disorders in pregnancy: a protocol for systematic review and meta-analysis. BMJ Open 2021;11:e046005. doi:10.1136/ bmjopen-2020-046005

- Prepublication history and additional material for this paper is available online. To view these files, please visit the journal online (http://dx.doi.org/10. 1136/bmjopen-2020-046005)

Received 20 0ctober 2020 Revised 23 March 2021 Accepted 24 March 2021

\section{Check for updates}

(c) Author(s) (or their employer(s)) 2021. Re-use permitted under CC BY-NC. No commercial re-use. See rights and permissions. Published by BMJ.

For numbered affiliations see end of article.

\section{Correspondence to} Dr Anne-Marie Côté; Anne-Marie.Cote@Usherbrooke. ca

\section{ABSTRACT}

Introduction Preeclampsia is an important cause of maternal and fetal morbidity and mortality. Although the diagnostic and prognostic values of circulating placental growth factor (PIGF) have been extensively studied, urinary PIGF represents an excellent alternative to facilitate sample collection in the follow-up of pregnant women. The aim of this study is to determine whether urinary PIGF levels throughout pregnancy can predict severe maternal, fetal/placental and neonatal complications in women with hypertensive disorders in pregnancy.

Methods and analysis Studies that evaluated pregnant women with hypertensive disorders and at least one measurement of urinary PIGF will be included. Studies that measure urinary PIGF after the occurrence of the complications will be excluded. The main outcome will consist of severe maternal complications in women with hypertensive disorders in pregnancy. Secondary outcomes will consist of severe fetal/placental and neonatal complications as defined by the International Collaboration to Harmonize Outcomes for Pre-eclampsia. Prospective cohort studies and case-controls studies reporting original data will be included. Studies will be identified by searching MEDLINE and SCOPUS databases. The first literature search was conducted on 2 March 2020, and another search will be performed before analyses. All eligible studies will be assessed for risk of bias with a standardised 10 -items study quality assessment tool adapted from the Study Quality Assessment tools developed by the National Institutes of Health $(\mathrm{NIH})$. Summary of ORs and $95 \%$ Cls will be reported to evaluate the association between urinary PIGF levels and hypertensive disorders in pregnancy and its complications. A random-effect meta-analysis will also be performed. Ethics and dissemination Review by an ethics committee will not be required for this systematic review. This study will follow the Preferred Reporting Items for Systematic Reviews and Meta-Analyses guidelines and will be submitted for publication in a peer-reviewed journal as well as for presentation at conferences targeting different stakeholders, including researchers, physicians and patients.

PROSPERO registration number CRD42020186313.

\section{Strengths and limitations of this study}

- This systematic review and meta-analysis will offer a synthesis and a comprehensive understanding of the work done to this day on urinary testing of placental growth factor (PIGF) for prediction of outcomes in hypertensive disorders in pregnancy.

- This prospectively registered study will be rigorously conducted with the contributions of all authors sharing expertise in methodology and context.

- A small number of studies and the heterogeneity of the data may be a limitation of this study.

- This study may identify gaps in knowledge that could be addressed by future studies aimed at investigating the use of urinary PIGF in pregnancy.

\section{INTRODUCTION}

Preeclampsia is the most severe hypertensive disorder in pregnancy occurring in $2 \%-5 \%$ of all pregnancies around the globe. ${ }^{1}$ It is a leading cause of fetal and maternal morbidity and mortality and may cause severe complications. ${ }^{1}$ To help combine and compare the results of research on preeclampsia, the International Collaboration to Harmonize Outcomes for Pre-eclampsia (iHOPE) established a core outcome set that defines the maternal and fetal complications of preeclampsia. $^{2}$

Even if the underlying causes of preeclampsia remain unknown, preeclampsia may be caused by a defect of circulating angiogenic factors leading to endothelial cells dysfunction. ${ }^{34}$ As such, it has been shown that women diagnosed with preeclampsia present increased circulating blood levels of the antiangiogenic factor soluble fms-like tyrosine kinase 1 (sFlt- 1$){ }^{5}$ sFlt-1 normally binds angiogenic factors such as the vascular endothelial growth factor (VEGF) as well as the placental 
growth factor (PIGF). Increased circulating levels of sFlt-1 lead to a reduction in free PIGF and VEGF and prevents their action on endothelial cells generating endothelial dysfunction. ${ }^{6}$

Previous observational retrospective study suggested that the maternal blood levels of angiogenic and antiangiogenic factors may be altered as soon as 5 weeks prior to the clinical manifestation of preeclampsia, ${ }^{7}$ or even as soon as the first trimester in pregnancy. ${ }^{5}$ Likewise, several investigators conducted clinical studies to evaluate the prognostic and diagnostic values of circulating angiogenic factors in hypertensive pregnant women. ${ }^{8-10}$ Circulating levels of PIGF can predict the occurrence of preeclampsia and its complications. It has been shown that circulating levels of PIGF are lower in patient with preeclampsia compared with healthy controlled patient $(61.3 \pm 28.1$ vs $122.4 \pm 81.0 \mathrm{pg} / \mathrm{ml} \quad(\mathrm{p}<0.001)) .{ }^{9}$ Current efforts have focused on the assessment of circulating levels of angiogenic factors. However, as these routine blood tests during pregnancy are time consuming, expensive and certainly uncomfortable to pregnant women, the development of a urinary test may be more convenient and acceptable.

Diagnostic and prognostic values of circulating angiogenic factors have been studied extensively in the past, but less is known about urinary PIGF diagnostic and prognostic values. PIGF is a low molecular weight protein of $\sim 30 \mathrm{kDa}^{11}$, which is filtered by the kidneys. Urinary levels of this protein highly correlate with its circulating blood levels $(r=0.934),{ }^{12}$ thus making urinary PlGF an interesting alternative for routine testing. This systematic review and meta-analysis will offer a synthesis and understanding of the work done to this day on urinary testing of PIGF for prediction of outcomes in hypertensive disorders in pregnancy.

\section{OBJECTIVE}

The main objective of this study is to determine whether urinary PIGF levels throughout pregnancy can predict severe maternal, fetal/placental and neonatal complications in women with hypertensive disorders in pregnancy.

\section{METHOD AND ANALYSIS \\ Protocol and registration}

The Preferred Reporting Items for Systematic Review and Meta-Analysis Protocols ${ }^{13}$ were followed for the elaboration of this protocol (see online supplemental file 1). This systematic review was registered prospectively.

\section{Participants}

The review will target studies that include pregnant women with hypertensive disorders in pregnancy and at least one measurement of urinary PlGF during pregnancy. Studies with measurements performed after the occurrence of the complications and postpartum will not be included. No exclusion criteria based on underlying maternal medical conditions will be applied.

\section{Outcome measurement}

Patient and public involvement

A panel of four experts in maternal and fetal medicine as well as a patient with lived experience of preeclampsia were consulted to determine and prioritise the outcomes of the study. A medical student was involved in reviewing the protocol for completeness and to ensure its clarity to non-expert readers.

The main outcome will consist of severe maternal complications in women with hypertensive disorders in pregnancy (as defined below). Secondary outcomes will consist of severe fetal/placental, neonatal complications.

The core outcomes set for preeclampsia developed by the $\mathrm{iHOPE}^{2}$ will be used as follow:

1. Maternal core outcomes as: maternal mortality, severe morbidity (eg, eclampsia, stroke, cortical blindness, retinal detachment, pulmonary oedema, acute kidney injury, liver capsule haematoma or rupture, placental abruption, postpartum haemorrhage, raised liver enzymes, low platelets, admission to intensive care unit required, intubation and mechanical ventilation (not for childbirth)).

2. Offspring outcomes as: stillbirth, gestational age at delivery (defined as delivery $<37$ weeks of gestation), birth weight, small-for-gestational-age (defined as a $\leq 10$ growth centile), neonatal mortality, neonatal seizures, admission to neonatal unit required, respiratory support.

Considering the heterogeneity of the outcomes used in the different studies, we will also examine the outcomes defined in the Pre-eclampsia Integrated Estimate of RiSk ${ }^{14}$ study or their equivalent, including hemolysis, elevated liver enzymes and a low platelet count syndrome, disseminated intravascular coagulation, intrauterine growth restriction, abnormal fetal Doppler and oligohydramnios. Definitions of small-for-gestational-age will include the Gordijn and Beune definition as per the Delphi procedure. ${ }^{15}$ This suggestion was put forth by our expert panel for determination and prioritisation of outcomes for also applying more stringent criteria for placental insufficiency.

The following definitions will be used to classify hypertensive disorders in pregnancy ${ }^{16}$ :

- Gestational hypertension will be defined as de novo persistent hypertension after 20 weeks of pregnancy (blood pressure $\geq 140 \mathrm{~mm} \mathrm{Hg}$ systolic or $\geq 90 \mathrm{~mm} \mathrm{Hg}$ diastolic).

- Chronic hypertension will be defined as hypertension (blood pressure $\geq 140 \mathrm{~mm} \mathrm{Hg}$ systolic or $\geq 90 \mathrm{~mm} \mathrm{Hg}$ diastolic) predated to pregnancy or discovered before 20 weeks of gestation.

- Preeclampsia will be defined as gestational hypertension accompanied by one or more new-onset conditions among proteinuria as a protein/creatinine ratio $\geq 0.3 \mathrm{~g} / \mathrm{g}$, protein $\geq 1 \mathrm{~g} / \mathrm{L}, 24$ hours collection $\geq 0.3 \mathrm{~g}$ / 
day or one dipstick measurement $\geq 1+$, acute kidney injury, liver involvement, neurological complications, haematological complications or uteroplacental dysfunction.

- Superimposed preeclampsia will be defined as any of the maternal organ dysfunction of preeclampsia in a woman with chronic hypertension.

\section{Type of studies}

Prospective cohort studies and case-controls studies reporting original data will be included in this review. As the main objective is to search for a predictor, prospective studies are best designed for that purpose and though some cross-sectional studies may fulfil the requirement, it will be very difficult for retrospective studies to serve data for a predictive analysis. Literature reviews, case studies and case reports, letter to the editors, comments on article and editorials will be excluded. Study focusing only on animal research, on PIGF quantification in serum or plasma or study unrelated to pregnancy will also be excluded. Only studies published in French or in English will be included. No time restriction will be applied.

\section{Search strategy}

MEDLINE and SCOPUS will be searched for the systematic review and another search will be planned closer to the publication of the results. The first search was conducted on 2 March 2020, and another search will be performed before the analyses. The reference lists of the included studies will also be reviewed manually. The complete search strategy was reviewed and validated by a librarian and is provided as online supplemental file 2 .

\section{Study records}

Study selection

Inclusion criteria were fixed by two reviewers (CF, A-MC). Studies will be imported in Zotero V.5.0.89 and duplicates will be removed. Studies will be independently screened by two reviewers according to title and abstract (CF, JM). Full text will be assessed if the sorting is still unclear. Disagreement between the two reviewers will be resolved with the opinion of a third reviewer (A-MC). The sorting will be compiled in an Excel spreadsheet.

\section{Data collection}

The data will be extracted independently in duplicate by two reviewers using a prepiloted standardised data extraction form. The following data will be extracted from the publications: author, publication year, study group, laboratory, aim of the study, population, gestational age, definition of hypertensive disorders in pregnancy, preeclampsia, identified outcomes, laboratory method for determination of urinary PIGF, the brand of the PIGF assay kit, urinary PIGF results and proposed cut-off, if the urinary PIGF results were standardised according to the urinary creatinine, summary test characteristics (sensitivity, specificity, likelihood ratios, area under the receiver-operating characteristic (ROC) curve), OR. Disagreement between two reviewers will be resolved with the opinion of a third reviewer (A-MC). Missing data will be reported as well and the quality of the article will be assessed accordingly. The data will be compiled in an Excel spreadsheet.

\section{Risk of bias assessment}

A standardised 10-items study quality assessment tool adapted from the Study Quality Assessment tools developed by the National Institutes of Health (NIH) (https:/ / www.nhlbi.nih.gov/health-topics/study-quality-assessment-tools) will be used by two independent observers. Each study included will be classified as either good, fair or poor quality. The strength of evidence between studies will be determine using the Grading of Recommendations, Assessment, Development and Evaluations (GRADE) system. ${ }^{17}$

\section{Summarising data}

Primary analysis will be conducted using only urinary PlGF standardised for urinary creatinine. Summary of OR and 95\% CIs will be reported to evaluate the association between urinary PIGF levels and hypertensive disorders in pregnancy and its complications. If there is a sufficient number of studies, we will pool the results with a random-effects meta-analysis due to a priori concerns that not all included studies will assess comparable populations. Pooled effect sizes will be obtained using Mantel-Haenszel method, a more reliable approach than inverse-variance to determine study weights. Since a small number of studies presenting OR is expected, we decided to focus our analyses on effect size. However, studies presenting Relative Risk (RR) and Likelihood ratio (LR) could be considered for potential comparisons. Furthermore, if the number of valid studies allows it, hierarchical summary receiver-operating characteristic models will be presented as pooled accuracy measures and 95\% CIs. Summary of sensitivity and specificity with $95 \%$ CIs will also be reported. For both pooled effects presented, heterogeneity will be assessed by Higgins' and Thomson's I ${ }^{2}$ index. Analyses will be performed using the meta package from R Software V.4.0.0 (R Core Team (2020). R Foundation for Statistical Computing, Vienna, Austria).

\section{Prespecified subgroup analyses}

We will consider clinical heterogeneity and if there is sufficient power, we will conduct subgroup analyses according to type of hypertensive disorder in pregnancy (preeclampsia de novo vs superimposed preeclampsia, preterm preeclampsia $<37$ and $<34$ weeks), trimester in pregnancy at urinary collection and method of PIGF measurement, as well as maternal medical conditions. Variables of interest were chosen for subgroup analyses considering their clinical relevance in leading to a potential gain of information. Heterogeneity will be quantify using $\mathrm{I}^{2}$ index and a $\chi^{2}$ test will be performed to assess for homogeneity between subgroups with statistical significance at $5 \%$. 


\section{Sensitivity analyses}

Sensitivity analyses will be conducted by study epoch and study quality if numbers are sufficient.

\section{Assessment of reporting bias}

Considering that studies with inconclusive or nonsignificant results often remain unpublished, we will assess reporting bias using a funnel plot if at least 10 studies are included in the meta-analysis. ${ }^{18}$ In the absence of publication bias, all studies will lie symmetrically around the calculated pooled odd ratios. Since the interpretation of the funnel plot is subjective, arcsine test for dichotomous outcomes measured as ORs will be presented to quantifies asymmetry considering a statistical significance of $5 \% .{ }^{19}$

\section{Interpretation of results}

All authors will contribute to the interpretation of the results with their respective input of expertise for methodology and statistical analyses (M-CB, SL-P) and for content and context (CF, JM, A-MC, M-ER-L) and a virtual meeting will be planned to discuss and debate the results and reach consensus for interpretation and presentation of results. We will assess the quality of evidence using the GRADE framework. ${ }^{17}$

\section{Protocol amendments}

Any amendments to the protocol will be reported, justified and dated.

\section{Ethics and dissemination}

Review by an ethics committee will not be required since no data from participants will be collected. The results of this systematic review and meta-analysis will follow the Preferred Reporting Items for Systematic Reviews and Meta-Analyses guidelines ${ }^{20}$ and will be submitted for publication in a peer-reviewed journal. The results will also be submitted for presentation at conferences targeting different stakeholders, including researchers, physicians and patients.

\section{DISCUSSION}

Preeclampsia remains an important cause of maternal, fetal and neonatal mortality and morbidity. Testing for angiogenic factors can improve identification and management of women with hypertensive disorders in pregnancy. Urinary testing is easier to obtain than blood samples and as a non-invasive test, it is more acceptable to most women during antenatal care. Thus, urinary PIGF represents a great alternative in the evaluation of pregnant women and to facilitate longitudinal follow-up. Moreover, serial urinary samples may allow for better interpretation of biomarker and clinical trajectory of women at risk or developing an hypertensive disorder in pregnancy. Finally, in low-resource settings and remote areas, as well as for telemonitoring, urinary testing for PlGF may eventually be more accessible to guide management and medical decisions at lower costs.

\section{Strengths and limitations}

This prospectively registered study provides a search strategy, well-defined outcomes and analysis plan. The systematic review and meta-analysis will be rigorously conducted with the contributions of all authors sharing expertise in methodology and context. The GRADE framework will be used to report our results. Considering the interest for urinary PIGF is recent, the small numbers of studies and the heterogeneity of the data may be a limitation of this study. However, this systematic review may identify future areas of study on the use of urinary PlGF in pregnancy.

\section{Author affiliations}

${ }^{1}$ Division of Nephrology, Department of Medicine, Faculty of Medicine and Health Sciences, University of Sherbrooke, Sherbrooke, Québec, Canada

${ }^{2}$ Centre de Recherche du Centre Hospitalier Universitaire de Sherbrooke,

Sherbrooke, Québec, Canada

${ }^{3}$ Department of Medicine, Faculty of Medicine and Health Sciences, University of Sherbrooke, Sherbrooke, Québec, Canada

${ }^{4}$ Department of Obstetrics and Gynecology, Faculty of Medicine and Health Sciences, University of Sherbrooke, Sherbrooke, Québec, Canada

Acknowledgements We thank Dr Annabelle Cumyn, Dr Annie Ouellet, Dr Evelyne Rey and Dr Evelyne Raîche, expert panel in maternal and fetal medicine, MarieNoëlle Richard, patient with lived experience of preeclampsia, Josée Toulouse, librarian and Anne-Marie Lafaille-Hebert, medical student, for their contribution to the protocol. Dr Cumuy also kindly revised the manuscript for linguistic review.

Contributors All authors contributed to the preparation of the protocol, reviewed and approved the final version of the protocol. A-MC and CF conceived the research question. A-MC, CF, M-CB, JM and SL-P contributed to the methodological aspects and analysis sections of the protocol. M-ER-L contributed to expert content and outcomes definitions. A-MC is the guarantor of this review.

Funding $\mathrm{CF}$ is a recipient of a Postgraduate Scholarship from the Faculty of Medicine and Health Sciences of Université de Sherbrooke. A-MC's research program is supported by funding from the Canadian Institutes of Health Research Research (MFM-46445).

Competing interests None declared.

Patient consent for publication Not required.

Provenance and peer review Not commissioned; externally peer reviewed.

Supplemental material This content has been supplied by the author(s). It has not been vetted by BMJ Publishing Group Limited (BMJ) and may not have been peer-reviewed. Any opinions or recommendations discussed are solely those of the author(s) and are not endorsed by BMJ. BMJ disclaims all liability and responsibility arising from any reliance placed on the content. Where the content includes any translated material, BMJ does not warrant the accuracy and reliability of the translations (including but not limited to local regulations, clinical guidelines, terminology, drug names and drug dosages), and is not responsible for any error and/or omissions arising from translation and adaptation or otherwise.

Open access This is an open access article distributed in accordance with the Creative Commons Attribution Non Commercial (CC BY-NC 4.0) license, which permits others to distribute, remix, adapt, build upon this work non-commercially, and license their derivative works on different terms, provided the original work is properly cited, appropriate credit is given, any changes made indicated, and the use is non-commercial. See: http://creativecommons.org/licenses/by-nc/4.0/.

ORCID iD

Anne-Marie Côté http://orcid.org/0000-0002-1252-769X

\section{REFERENCES}

1 Phipps EA, Thadhani R, Benzing T, et al. Pre-Eclampsia: pathogenesis, novel diagnostics and therapies. Nat Rev Nephrol 2019;15:275-89. 
2 Duffy JMN, Cairns AE, Richards-Doran D. The International collaboration to Harmonise outcomes for pre-eclampsia (iHOPE). A core outcome set for pre-eclampsia research: an international consensus development study. Int J Obstet Gynaecol 2020;127:1516-26.

3 Maynard SE, Min J-Y, Merchan J, et al. Excess placental soluble FMS-like tyrosine kinase 1 (sFlt1) may contribute to endothelial dysfunction, hypertension, and proteinuria in preeclampsia. J Clin Invest 2003;111:649-58.

4 Tomimatsu T, Mimura K, Matsuzaki S, et al. Preeclampsia: maternal systemic vascular disorder caused by generalized endothelial dysfunction due to placental antiangiogenic factors. Int J Mol Sci 2019;20. doi:10.3390/ijms20174246. [Epub ahead of print: 30 Aug 2019].

5 Thadhani R, Mutter WP, Wolf M, et al. First trimester placental growth factor and soluble FMS-like tyrosine kinase 1 and risk for preeclampsia. J Clin Endocrinol Metab 2004;89:770-5.

6 Koga K, Osuga Y, Yoshino O, et al. Elevated serum soluble vascular endothelial growth factor receptor 1 (sVEGFR-1) levels in women with preeclampsia. J Clin Endocrinol Metab 2003;88:2348-51.

7 Levine RJ, Maynard SE, Qian C, et al. Circulating angiogenic factors and the risk of preeclampsia. N Engl J Med 2004;350:672-83.

8 Taylor RN, Grimwood J, Taylor RS, et al. Longitudinal serum concentrations of placental growth factor: evidence for abnormal placental angiogenesis in pathologic pregnancies. Am J Obstet Gynecol 2003;188:177-82.

9 Polliotti BM, Fry AG, Saller DN, et al. Second-Trimester maternal serum placental growth factor and vascular endothelial growth factor for predicting severe, early-onset preeclampsia. Obstet Gynecol 2003;101:1266-74.

10 Duhig KE, Myers J, Seed PT, et al. Placental growth factor testing to assess women with suspected pre-eclampsia: a multicentre, pragmatic, stepped-wedge cluster-randomised controlled trial. Lancet 2019;393:1807-18.

11 Tammela T, Enholm B, Alitalo K, et al. The biology of vascular endothelial growth factors. Cardiovasc Res 2005;65:550-63.

12 Tang P, Xu J, Xie B-J, et al. Use of serum and urinary soluble sFlt-1 and PIGF in the diagnosis of preeclampsia. Hypertens Pregnancy 2017;36:48-52.

13 Moher D, Shamseer L, Clarke M, et al. Preferred reporting items for systematic review and meta-analysis protocols (PRISMA-P) 2015 statement. Syst Rev 2015;4:1.

14 Ukah UV, Payne B, Hutcheon JA, et al. Assessment of the fullPIERS risk prediction model in women with early-onset preeclampsia. Hypertension 2018;71:659-65.

15 Gordijn SJ, Beune IM, Thilaganathan B, et al. Consensus definition of fetal growth restriction: a Delphi procedure. Ultrasound Obstet Gynecol 2016;48:333-9.

16 Brown MA, Magee LA, Kenny LC, et al. The hypertensive disorders of pregnancy: ISSHP classification, diagnosis \& management recommendations for international practice. Pregnancy Hypertens 2018;13:291-310.

17 Guyatt GH, Oxman AD, Kunz R, et al. GRADE guidelines: 8. Rating the quality of evidence--indirectness. $J$ Clin Epidemiol 2011;64:1303-10.

18 Begg CB, Mazumdar M. Operating characteristics of a RANK correlation test for publication bias. Biometrics 1994;50:1088-101.

19 Rücker G, Schwarzer G, Carpenter J. Arcsine test for publication bias in meta-analyses with binary outcomes. Stat Med 2008;27:746-63.

20 Moher D, Liberati A, Tetzlaff J, et al. Preferred reporting items for systematic reviews and meta-analyses: the PRISMA statement. $J$ Clin Epidemiol 2009;62:1006-12. 